\title{
Effect of Temperature on Optical Properties of Vegetable Oils Using UV-Vis and Laser Fluorescence Spectroscopy
}

\author{
Mohammad E. Khosroshahi',2 \\ ${ }^{1}$ Nanobiophotonics \& Biomedical Research Lab., MIS Electronics, Richmond Hill, Canada \\ ${ }^{2}$ Department of Mechanical \& Industrial Engineering, University of Toronto, Toronto, Canada \\ Email:khosrom@mie.utoronto.ca
}

How to cite this paper: Khosroshahi, M.E. (2018) Effect of Temperature on Optical Properties of Vegetable Oils Using UV-Vis and Laser Fluorescence Spectroscopy. Optics and Photonics Journal, 8, 247-263. https://doi.org/10.4236/opj.2018.87021

Received: June 5, 2018

Accepted: July 20, 2018

Published: July 23, 2018

Copyright $\odot 2018$ by author and Scientific Research Publishing Inc. This work is licensed under the Creative Commons Attribution International License (CC BY 4.0).

http://creativecommons.org/licenses/by/4.0/

\section{c) (i) Open Access}

\begin{abstract}
UV-Vis absorption and fluorescence spectroscopy are used to test the quality and changes in the composition of extra virgin olive oil (EVOO) and canola oil (CO) with temperature. The increase of temperature caused a change in the molecular structures of both types of oils seen as a gradual decrease of intensity amplitudes of absorption and fluorescence signals. A significant alteration occurred at $\approx 200^{\circ} \mathrm{C}$ where almost the main spectra of pheophytin-a, b, carotenoids, lutein and vitamin E in EVOO and linoleic acid and oleic acid in CO disappeared. An independent experiment showed the output of laser changes linearly with the input in oil at constant temperature (i.e., room temperature) where the transmission values of $\approx 33 \%$ and $\approx 75 \%$ are determined for EVOO and $\mathrm{CO}$ respectively. However, the transmission through a heated oil exhibited a non-linear behaviour which indicates the molecular optical response to thermal changes. The effect of storage time and adulteration of oils were also evaluated.
\end{abstract}

\section{Keywords}

Extra Virgin Oil, Canola, Temperature, Optical Properties, UV-Vis

Absorption, Fluorescence Spectroscopy, Laser

\section{Introduction}

There is a wide range of vegetable oils which are used for variety of daily applications such as cooking, cosmetics and pharmaceuticals. For example, olive and canola oils are considered mostly monounsaturated fat, while corn and soybean oils contain mainly polyunsaturated fat (i.e., more than one double bond) and 
coconut oil is predominantly saturated fat (i.e., no double bond in the molecule). For the purpose of this research an EVOO and $\mathrm{CO}$ are selected for comparison. Olive oil is a complex compound constitutes of fatty acids $\mathrm{CH}_{3}\left(\mathrm{CH}_{2}\right) \mathrm{nCOOH}$ such as monounsaturated oleic $(\approx 83 \%)$ and polyunsaturated linoleic acid $(\approx 21 \%)$, vitamins (e.g. vitamin $\mathrm{E}$ ), water soluble components and used throughout the world particularly in Mediterranean countries. Fatty acid, is a carboxylic acid (R-COOH) consisting of a long hydrocarbon chain, with a terminal carboxyl group. The composition of olive oil varies with the cultivar, altitude, time of harvest and extraction process., i.e., cold-pressed or refined [1] [2]. If the olive oil is made by pressing and did not undergo any of the industrial processes used to make "refined" oils such as canola, sunflower, soybean, it is referred to as "virgin" olive oil (VOO). The lower grades of olive oils are labeled as "Pure", "Light", or simply "Olive Oil".

Generally, the health-related beneficial properties of olive oil for cardiovascular disease and cancer prevention (e.g. colon cancer) are mainly attributed to its major components such as omega- 3 and omega- 6 fatty acids, vitamins (e.g., a-tocopherol, an antioxidant form of vitamin E) and strong antioxidant substances such as polyphenols [3] [4]. There for, VOO demonstrates high resistance to oxidation in comparison to other vegetable oils. An EVOO is the least processed form of olive oil with extra monounsaturated fatty acids than the other forms and is required to have no more than $0.8 \%$ free acidity, which improves its favorable flavor characteristics. The oxidative stability of EVOO correlates with the concentration of hydrophilic phenolic compounds and another significant minor component is $\alpha$-tocopherol, which protects the oil from oxidation at higher temperatures [5] [6]. Canola oil (CO) produced in Canada is obtained from the seeds of Brassica napus and Brassica rapa. These cultivars are low in erucic acid and glucosinolates and have different chemical and physical properties compared to high erucic acid rapeseed oil. The main composition of canola oil is: linolenic, linoleic, oleic, palmitic, steric, high oleic canola and low linolenic canola. Apart from heating, other parameters which directly affect the oil quality are light and the storage period. For these reasons they are more expensive due to the arduous tasks involved during the cultivation of olive trees, the harvesting and the extraction processes. This is normally the main reason for their adulteration with other edible oils of lower commercial value [7] [8]. Consequently, a rapid, inexpensive and non-invasive analytical technique would be desirable particularly for on-line applications. Considerable amount of research and information is available in literature using different techniques including high-performance liquid chromatography (HPLC) [9], Fourier-transform infra red spectroscopy (FTIR) [10], mass spectroscopy (MS) [11], nuclear magnetic resonance (MNR) [12], inductively coupled plasma optical emission spectroscopy (ICP-OES) [13], Raman spectroscopy [14] and optical spectroscopy such as absorption and fluorescence spectroscopy [15] [16].

Briefly, fluorescence as a kind of photoluminescence is based on the excitation of molecules from the ground state by absorption of radiation with an energy 
corresponding to the energy difference between the ground and excited states of a given fluorophore. The internal conversion and the vibrational relaxation are the two responsible processes for the emitted photon having lower energy than the incident photon. This shift to longer wavelength is known as Stokes shift, which is essential for sensitivity of fluorescence detection because it allows effective separation of the fluorescence emission signal from Rayleigh-scattered excitation singlet. The lifetime is about nanosecond.

It is noteworthy that laser-induced fluorescence spectroscopy (LIFS) has number of advantages that can be utilized to compensate some of the absorption spectroscopy (AS) where it generally indicates the presence of dyes and pigments. For example, in LIFS every molecule has its own optical signature exhibited as an emission due to absorption and excitation by a specific wavelength. This is even enhanced further if the wavelength is monochromatic and coherent such as a laser which can set to operate at only one wavelength only. Therefore, the molecular response would be very much selective indeed. This higher selectivity with lower background noise is counted as a major advantage of LIFS over typical AS. Also, LIF has higher sensitivity in contrast to AS where the emitted photons are detected against a low background. The sensitivity is about 100 1000 times higher than AS i.e., enabling the concentration measurement down to parts per billion levels [17]. Among other optical methods that are applied for biological and biomedical investigations, LIFS is one of the most widely spread spectroscopic methods which has been used for biomedical applications especially in diagnosis of cancer [18] [19]. T he purpose of this work is to describe the possibility of application of AS and LIFS to investigate the optical response of EVOO and canola oil after heating, storage time and adulteration.

\section{Materials and Methods}

Two EVOO in dark green colour glass, one fresh and other with 6 months storage time and a fresh canola oil were used for the experiment. The oils were poured in $10 \mathrm{~mm}$ cuvette without dilution for UV-Vis absorption spectroscopy (JENWAY-7205) between $200-900 \mathrm{~nm}$. Prior to measurements, a blank $10 \mathrm{~mm}$ cuvette filled with $\approx 80 \%$ distilled water was used for calibration and then cuvette filled with bubble-free oil was cleaned and placed inside the spectrometer with the clear side facing towards the light source. The samples were heated inside a glass beaker by a hot plate (Cole-palmer) close to spectrometer to reduce the cooling effects due to environment. The above procedure was repeated for the laser-induced fluorescence spectroscopy (LIFS) (Flame-T-XRI-ES-Ocean optics) using a $10 \mathrm{~mW} 405 \mathrm{~nm}$ diode laser pointer. A $10 \mathrm{~mW} 532 \mathrm{~nm}$ laser pointer was also used to compare and visualise the fluorescence with that of $405 \mathrm{~nm}$. For the independent transmission experiment, an optical fibre-coupled 4-channel laser (MCL-Thor Labs) was used at $406 \mathrm{~nm}$ with a variable out put power. A low level $1 \mathrm{~mW}$ He-Ne laser at $632 \mathrm{~nm}$ (Newport) was used to investigate the variation of transmission with temperature. The out put signal was detected by an avalanche 
photodiode power sensor (S121-C-Thor Labs) All LIFS experiments were performed in dark room at $20^{\circ} \mathrm{C}$ with the metal lid covering the cuvette to prevent from the background light noise.

\section{Results}

Figure 1(a) shows the absorption peaks of fresh EVOO at room temperature with multiple peaks where the 300 - 400 band provides some information about polyphenols antioxidants [16]. The absorption maxima located at positions around 425, 450 and $476 \mathrm{~nm}$ are due to presence of carotenoids, however, the $407 \mathrm{~nm}$ peak of pheophytin-a, which is a chemical compound that serves as the electron carrier in the electron transfer pathway of photosystem in plants can be easily overlapped by $424 \mathrm{~nm}$ peak of carotenoid. The $525 \mathrm{~nm}$ peak corresponds to vitamin $\mathrm{E}$ and chlorophyll band has two maxima positioned at 430 and 664 $\mathrm{nm}(\approx 670 \mathrm{~nm}$ in our case) respectively. The chlorophyll and carotenoids significantly affect the olive oil colour, which is an important factor for consumers. The greenish hues of VOO is due to chlorophyll pigments particularly pheophytin-a which is found in great amount in olive oils. The major yellow pigments of EVOO are explained by the presence of lutein and $\beta$-carotene. [20]. Lutein is one of two major carotenoids and like xanthophylls is found in high quantities in green leafy vegetables such as olive, spinach, and kale. The 450 and $476 \mathrm{~nm}$ peaks correspond to carotenoids. When the temperature gradually increased up to $50^{\circ} \mathrm{C}$ Figure 1 (b), no changes was observed at $425 \mathrm{~nm}$ but the peaks 450,476 and $670 \mathrm{~nm}$ slightly decreased. At $100^{\circ} \mathrm{C}$ Figure $1(\mathrm{c}), 450$ and $476 \mathrm{~nm}$ peaks almost disappeared and by further increasing the temperature to $200{ }^{\circ} \mathrm{C}$ Figure 1(d), all the peaks are now vanished except the dominant carotenoid peak at 425 $\mathrm{nm}$ and the $670 \mathrm{~nm}$ of chlorophyll which were decreased by $65 \%$ and $67 \%$ respectively.

Figure 2 represents the corresponding transmission curves for EVOO at room temperature (OR) and at $200^{\circ} \mathrm{C}(\mathrm{O}-200)$ respectively. Clearly, as temperature increases, the transmission also increases, which in this case corresponds to $\approx 30 \%$. This is investigated independently and will be discussed in the following sections.

As it can be seen in Figure 3, the corresponding absorption peaks of older EVOO show a significant difference compared with the fresh sample. The 425 $\mathrm{nm}$ peak has reduced considerably and the other maxima have almost vanished, however, the $670 \mathrm{~nm}$ peak shows no considerable change. A similar effect was observed when the temperature was increased and the amplitudes of corresponding peaks reduced by almost $80 \%$ and $50 \%$ for the above results respectively.

The experiment was repeated with $\mathrm{CO}$ and the results in Figure 4 show three distinct maxima at 285, 360 and $420 \mathrm{~nm}$ where the first one is thought to correspond to linoleic acid and the latter two to oleic acid respectively. It is interesting to note that the peaks amplitude did not change by increasing the temperature 


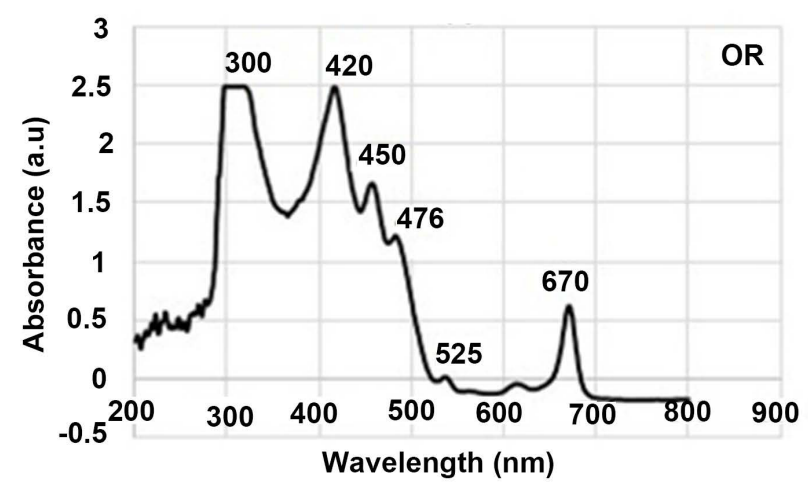

(a)

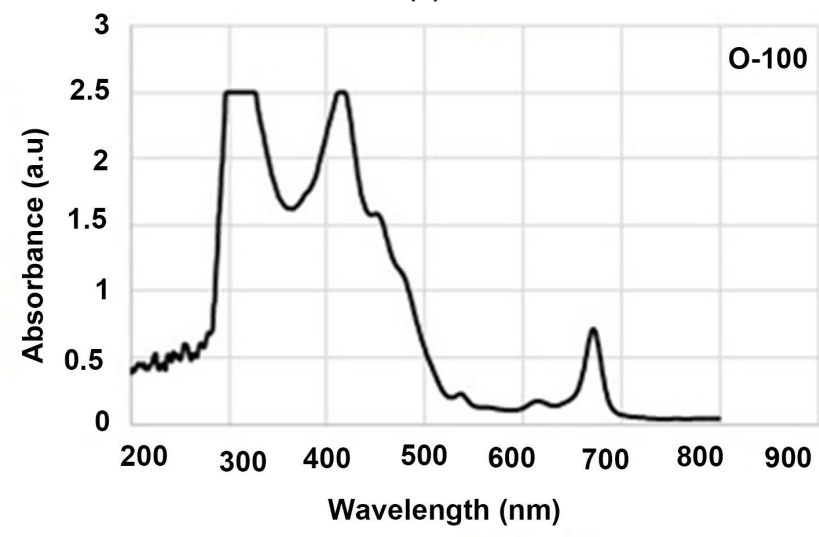

(c)

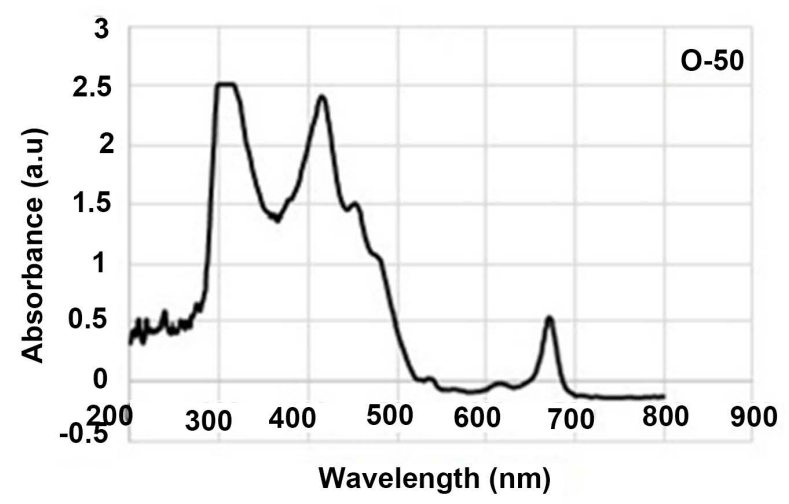

(b)

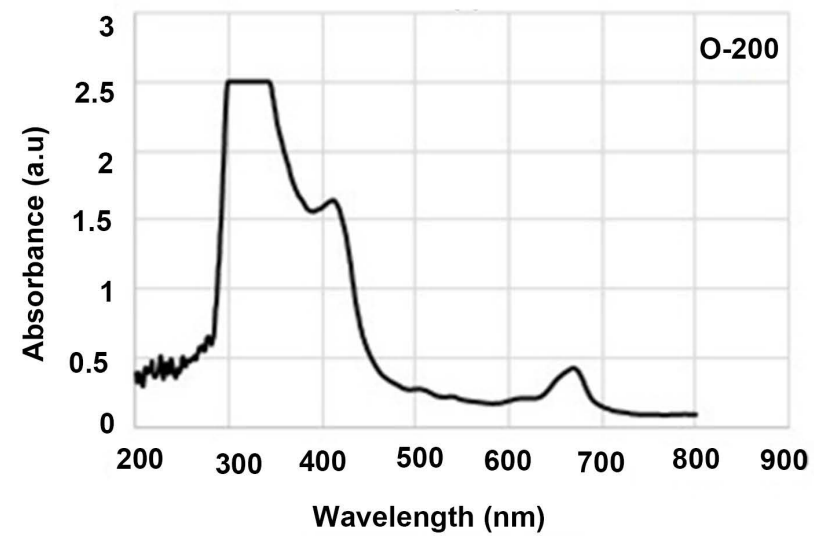

(d)

Figure 1. UV-Vis absorption spectroscopy of EVOO at different temperature.
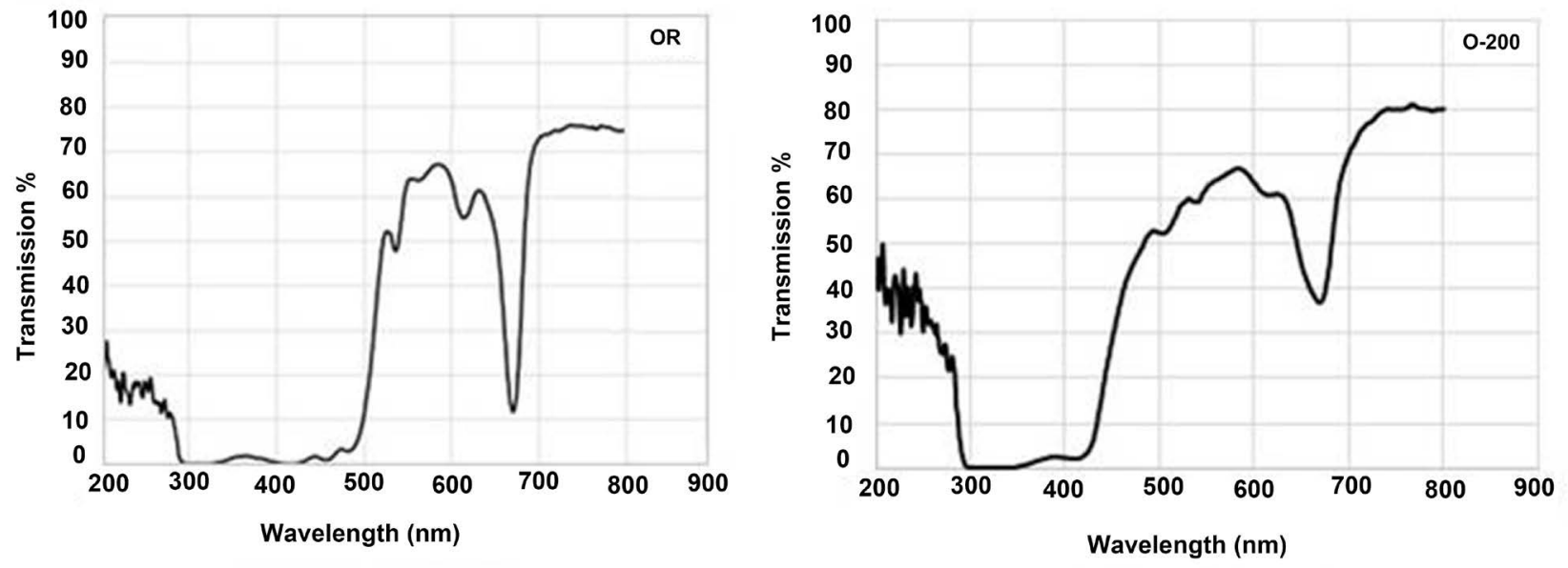

Figure 2. Transmission of EVOO at room temperature and $200^{\circ} \mathrm{C}$.

up to $50^{\circ} \mathrm{C}$ but it began to show a decreasing trend at $100^{\circ} \mathrm{C}$ where 285 and 360 $\mathrm{nm}$ lines almost reduced to its base line and the $420 \mathrm{~nm}$ reduced by about $20 \%$. At $200^{\circ} \mathrm{C}$, all lines are completely vanished and the $420 \mathrm{~nm}$ peak reduced by $50 \%$.

The transmission curves shown in Figure 5 demonstrates the fact that by increasing the temperature, transmission increases as expected similar to EVOO. 

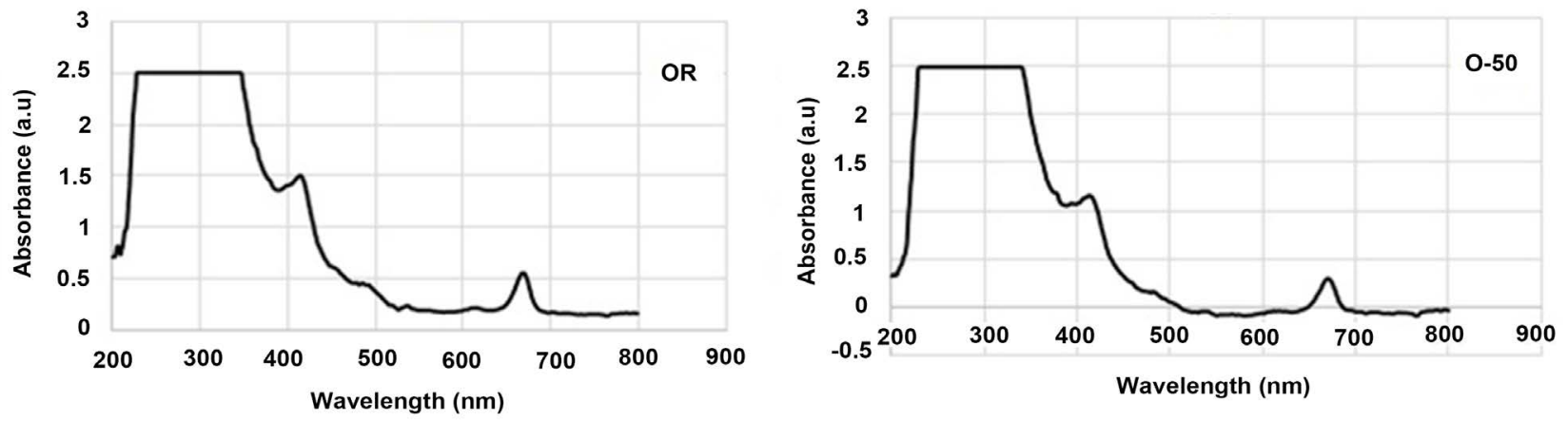

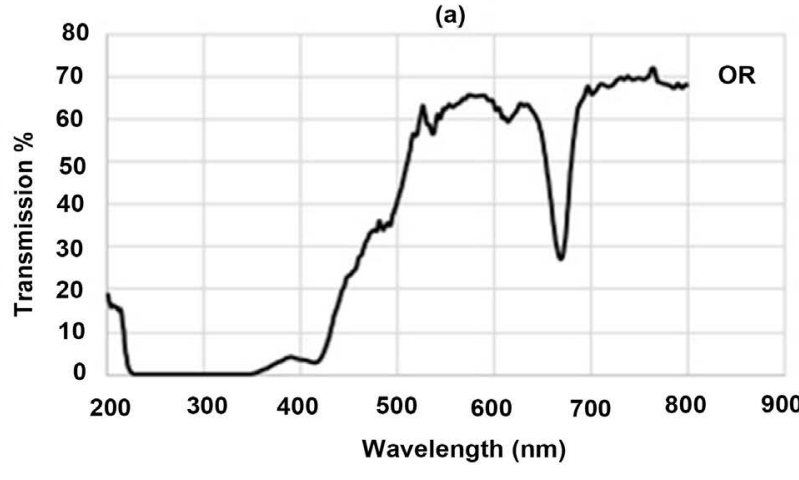

(c)

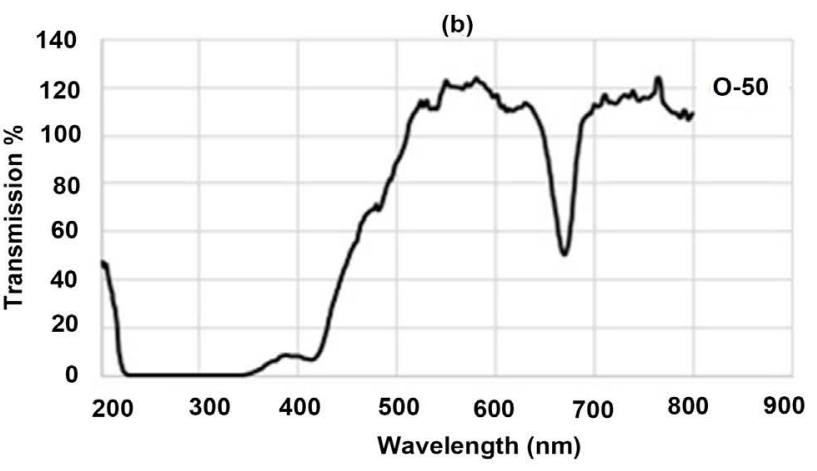

(d)

Figure 3. Comparison of absorbance and the corresponding transmission of aged EVOO at Room Temperature and $50^{\circ} \mathrm{C}$.
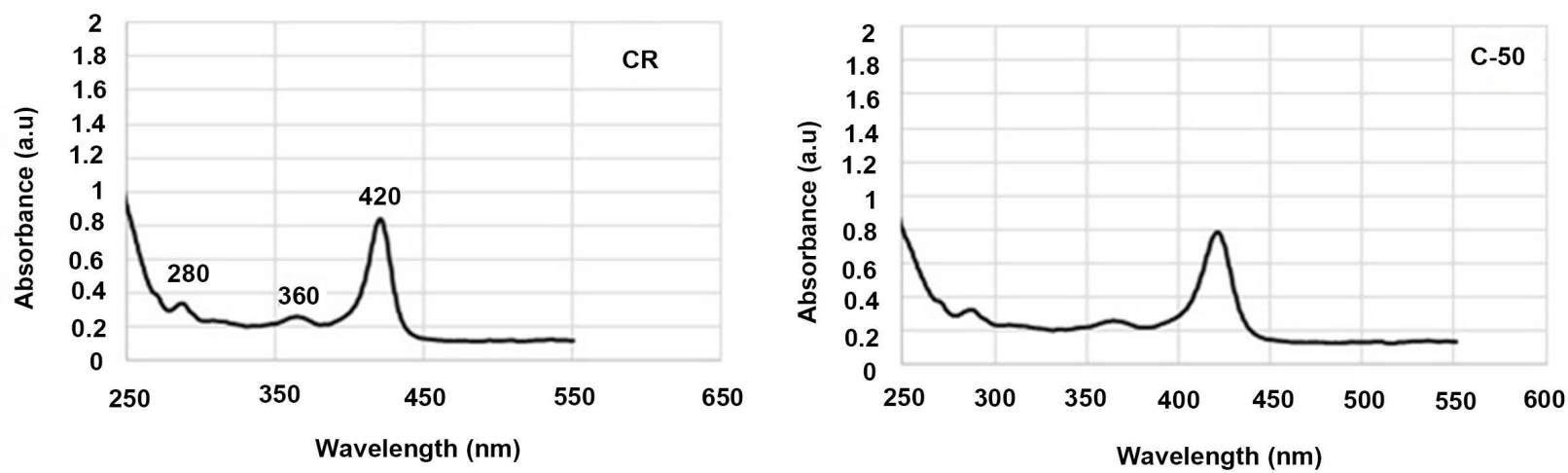

(a)

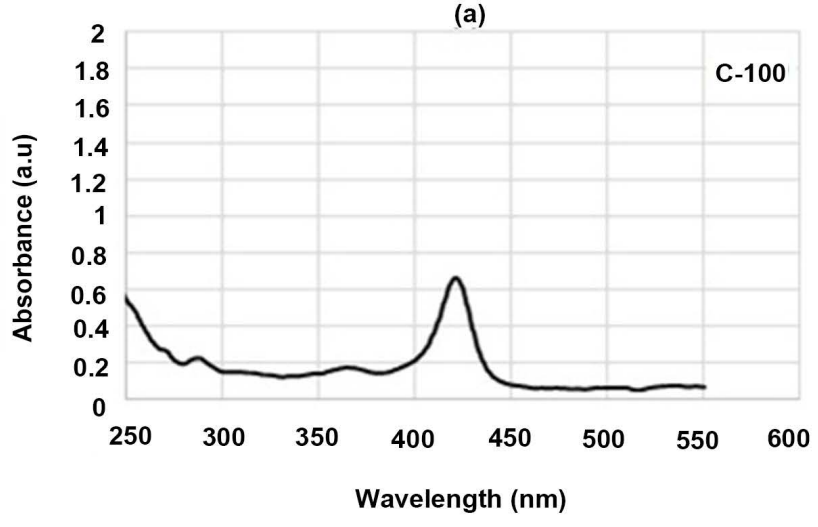

(c)

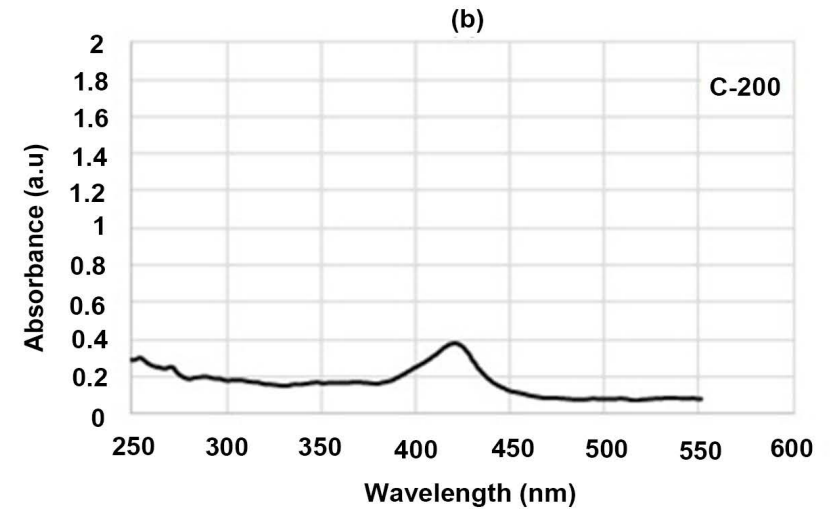

(d)

Figure 4. UV-vis absorbance of $\mathrm{CO}$ at different temperatures. 


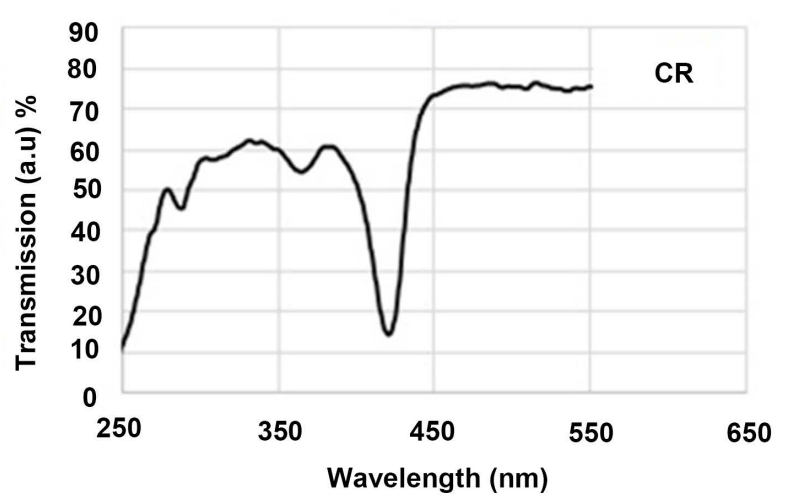

(a)

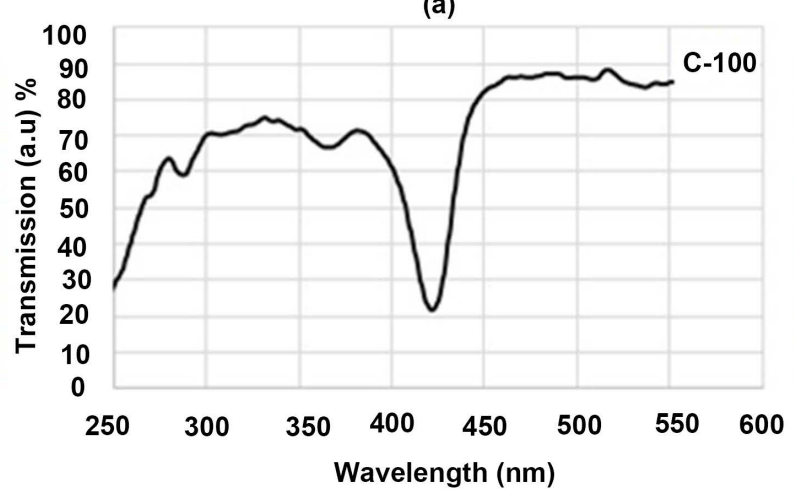

(c)

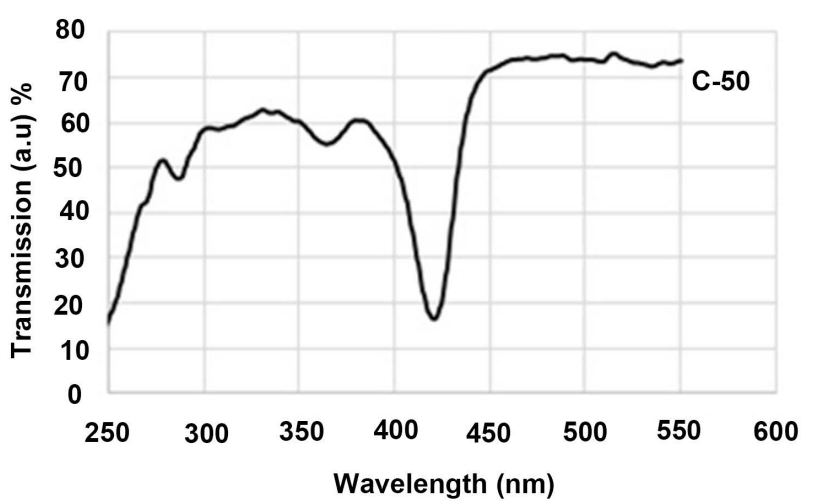

(b)

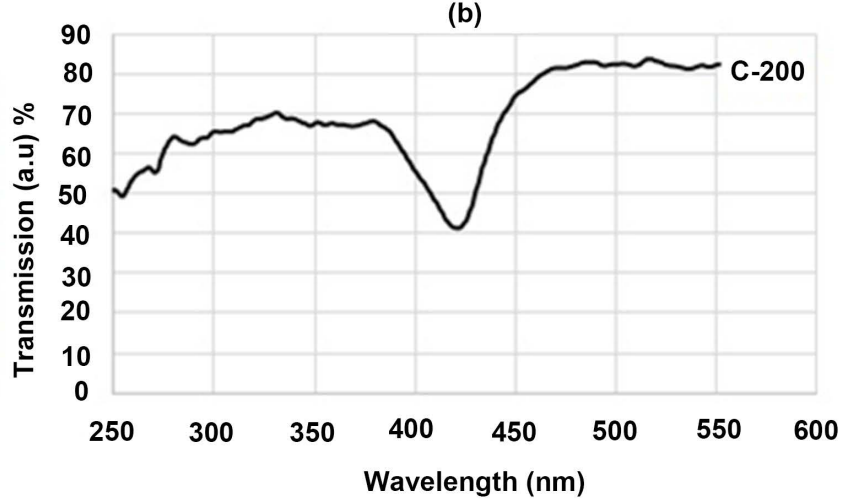

(d)

Figure 5. The corresponding transmission of $\mathrm{CO}$ at different temperatures.

Transmission of the dominant line at $420 \mathrm{~nm}$ increases from $\approx 15 \%$ at room temperature (CR) Figure 5(a) to $40 \%$ at $200^{\circ} \mathrm{C}(\mathrm{C}-200)$ in Figure 5(d). To test the adulteration effect, a mixture of EVOO and $\mathrm{CO}$ with equal ration $(50 \%$ $50 \%)$ was prepared and the experiment was repeated at room temperature. As seen in Figure 6(a), the EVOO showed its main characteristics but with much reduced amplitudes. Also, the line $360 \mathrm{~nm}$ of CO appeared in the curve. The corresponding transmission result is shown in Figure 6(b) where an increase of $\approx 20 \%$ occurs due to addition of higher transmissive properties of CO.

An independent experiment was performed to test the transmission properties of oils by using a $406 \mathrm{~nm}$ laser with variable out put power. The results in Figure 7 (a) indicate that for a given oil, the transmission increases by increasing the laser out put power and that it is higher for canola (75\%) compared to EVOO (33\%), which is explained in terms of optical behaviour of oils at molecular level in discussion section. Figure 7(b) illustrates an example of light transmission through EVOO and CO using 405 and $532 \mathrm{~nm}$ lasers in transverse and longitudinal directions. It is interesting to note that $532 \mathrm{~nm}$ wavelength shows higher transmission compared to $405 \mathrm{~nm}$ in EVOO. The beam broadening and divergence ar due to molecular collisions and thermal random Brownian motion during the diffusion process. A red fluorescence at $780 \mathrm{~nm}$ was observed using $532 \mathrm{~nm}$, Figure $7(\mathrm{c})$. 

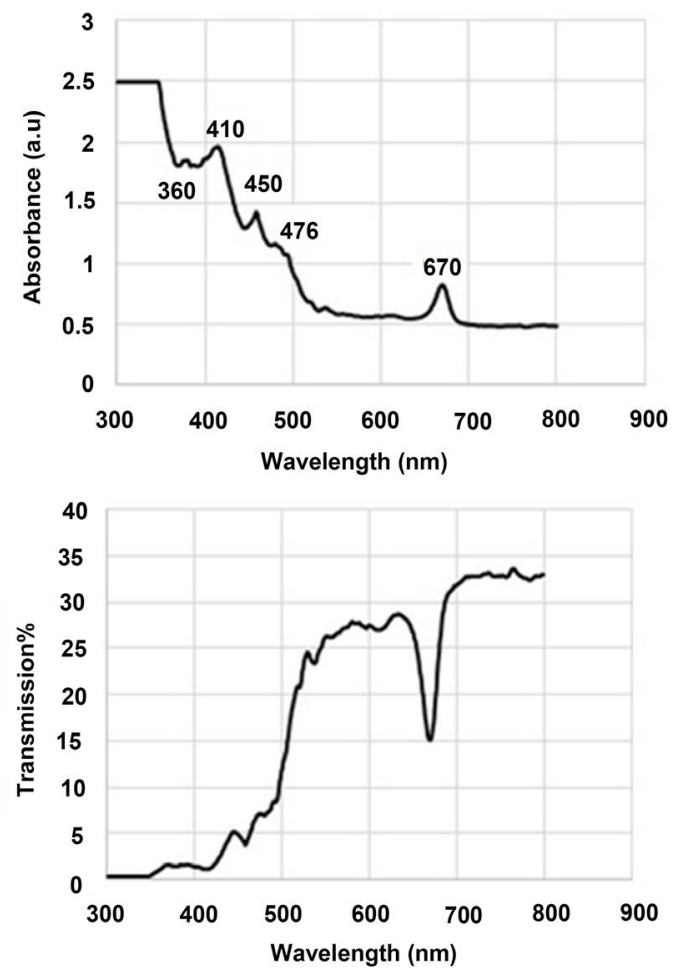

Figure 6. UV-Vis absorbance and the corresponding transmission of mixed EVOO and CO with 50-50\% ratio.

One way, light can interact with matter is through the process of light scattering, in which particles such as atoms or molecules in the path of a beam disperse the light in different directions. According to Rayleigh theory, particles with dimensions smaller than the light wavelength act as a point source elastic scattering where the intensity I of the scattered light depends on the square of particle volume $\mathrm{V}$ and distance $1 / r^{2}$ from the particles to the observer i.e.,

$$
I=\left(\frac{V^{2}}{r^{2} \lambda_{2}}\right) I_{0} f\left(n_{1}, n_{2}\right)
$$

where $f\left(n_{1}, n_{2}\right)$ is dimensionless function of the refractive indices of the particle and the environment. Assuming the small particles are identical in terms of their size, shape and orientation, the scattered light is symmetric about the particles. However, when the particles size increases, the wavelength dependence of the intensity of the scattered light decreases and the intensity distribution increases in the forward direction. In this case, the scattering phase function $\mathrm{p}$ for small angles is much higher than for all other angles, thus the mean cosine of the scattering g tends towards a value of unity. Therefore, higher the g value the more forward-peaked the scattering.

$$
g=\int_{4 \pi} p(\vartheta) \cos (\vartheta) \mathrm{d} \bar{s}^{\prime}
$$

where $\mathrm{d} \widehat{s}^{\prime}$ represents the photons travelling in direction $\mathrm{d} \widehat{s}$ are scattered into a new direction. It is noteworthy that the output signal is combination of 


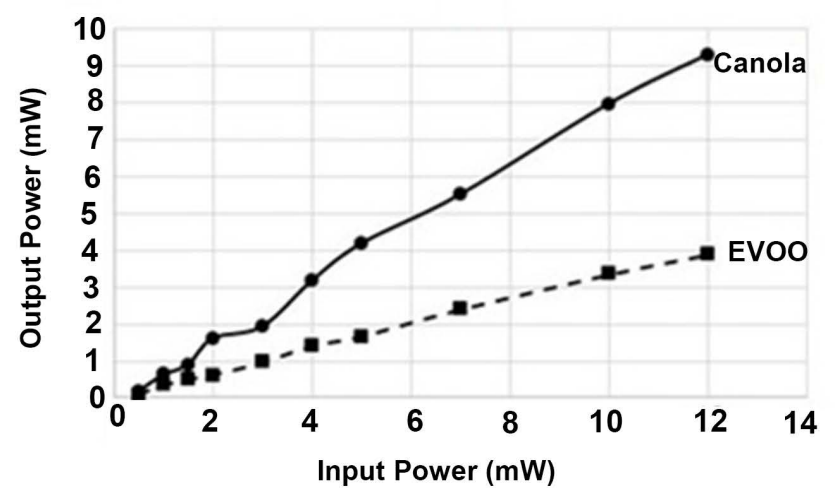

(a)

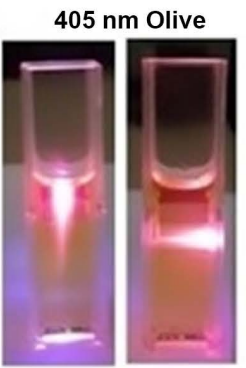

$532 \mathrm{~nm}$ Olive

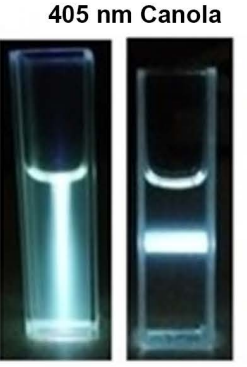

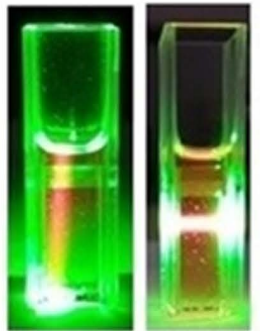

$532 \mathrm{~nm}$ Canola

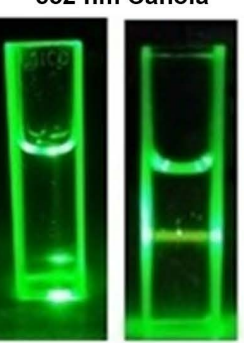

(b)

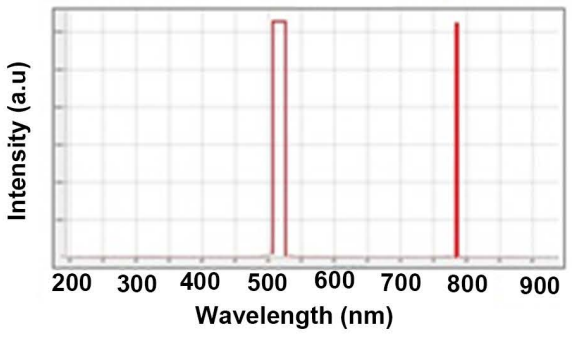

(c)

Figure 7. (a)Variation of $406 \mathrm{~nm}$ laser output power with input power for EVOO and CO at room temperature, (b) beam profile of 532 and $405 \mathrm{~nm}$ in transverse and parallel directions and the corresponding fluorescence of EVOO and CO at room temperature, (c) an example of 532-induced fluorescence response of EVOO.

transmitted and the forward scattered light at $180^{\circ}$. Based on the above explanation, it can be argued that the higher transmission by $\mathrm{CO}$ indicate the higher degree of combined forward scattering and the transmitted light as well as multiple scattering as this causes significant deviation from the spatial distribution and polarization of scattered light predicted bt Rayleigh scattering. Finally, in some cases, the disperded particles in colloidal suspensions may interact with each other and hence form ordered structures in the solution. This effectively can result in higher transmission of light within solution.

To gain a better understanding of thermal response of EVOO, an experiment was carried out using a low level He-Ne laser, Figure 8. The results represent the variation of laser out put with temperature and as it can be seen, the out put signal increases linearly up to $50^{\circ} \mathrm{C}$ where the turning point occurs and from that 


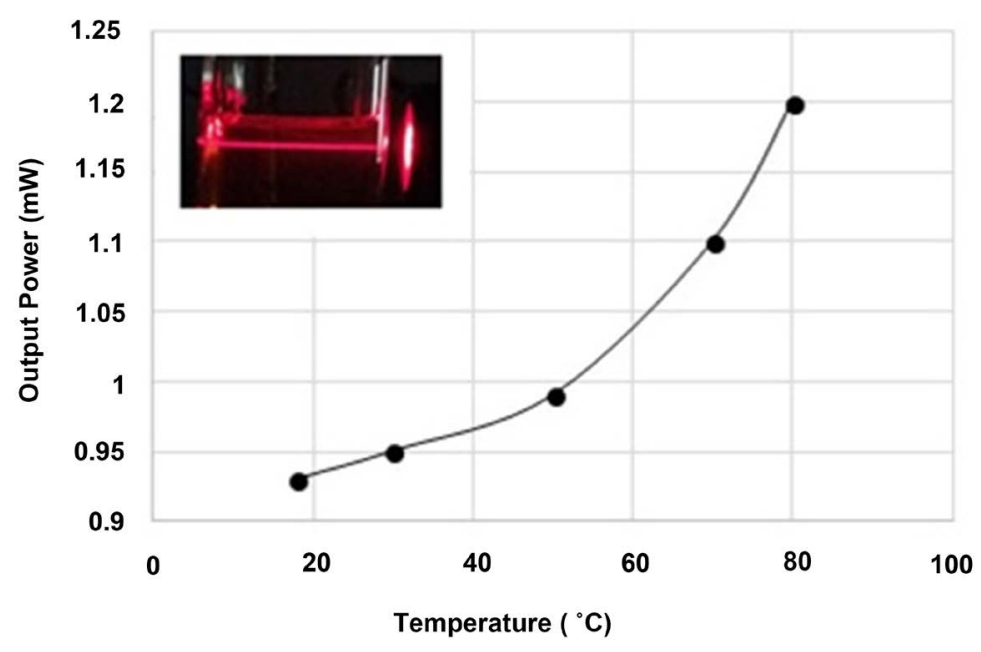

Figure 8. Variation of laser output with temperature for EVOO. The inset indicates the $\mathrm{He}-\mathrm{Ne}$ laser beam inside the oil.

point onward it continuous to increase non-linearly with temperature increase due to changes of oil chemical bonds and optical parameters such as change of refractive index.

Figure 9 represents the LIFS of EVOO at different temperatures. Clearly, at room temperature (OR) two distinct peaks at 525 and $660 \mathrm{~nm}$ are observed, which are likely related to Vitamin E and chlorophyll respectively. However, the intensity of fluorescence decreases with the increase of temperature such that only the $660 \mathrm{~nm}$ peak rapidly diminishes and almost disappears at O-200. The $525 \mathrm{~nm}$ peak only exhibits a change at $200^{\circ} \mathrm{C}$ where the amplitudes is reduced by about $30 \%$.

The experiment was repeated for $\mathrm{CO}$ as shown in Figure 10 and a major peak at $550 \mathrm{~nm}$ and a minor peak at $580 \mathrm{~nm}$ were observed (CR). Again, no considerable changes occurred until $100^{\circ} \mathrm{C}$ where the amplitudes of both peaks began to reduce. At $150^{\circ} \mathrm{C}$, the intensity decreased by $50 \%$ and at $200^{\circ} \mathrm{C}$ decreased even further down to about $37 \%$.

\section{Discussion}

This work describes the effects of temperature on composition and the quality of two widely used vegetable oils: EVOO and CO. Heating can be applied in different ways such as frying, boiling and microwave oven. Therefore, in the context of cooking, a variety of chemical changes are expected to occur depending on the nature of technique as well as the degree of heating and exposure time. These changes include chemical reactions, which in turn can be in the form hydrolysis, oxidation and polymerization all of which lead to possible loss of nutritional values and in some cases at long run causes a serious health problem due to release of free radicals namely cancer. Indeed, it has been shown [21] that microwave cooking of fatty foods at high temperature produces isomeration (i.e., formation of trans) of the double bonds of fatty acids. In a research, [22] used 

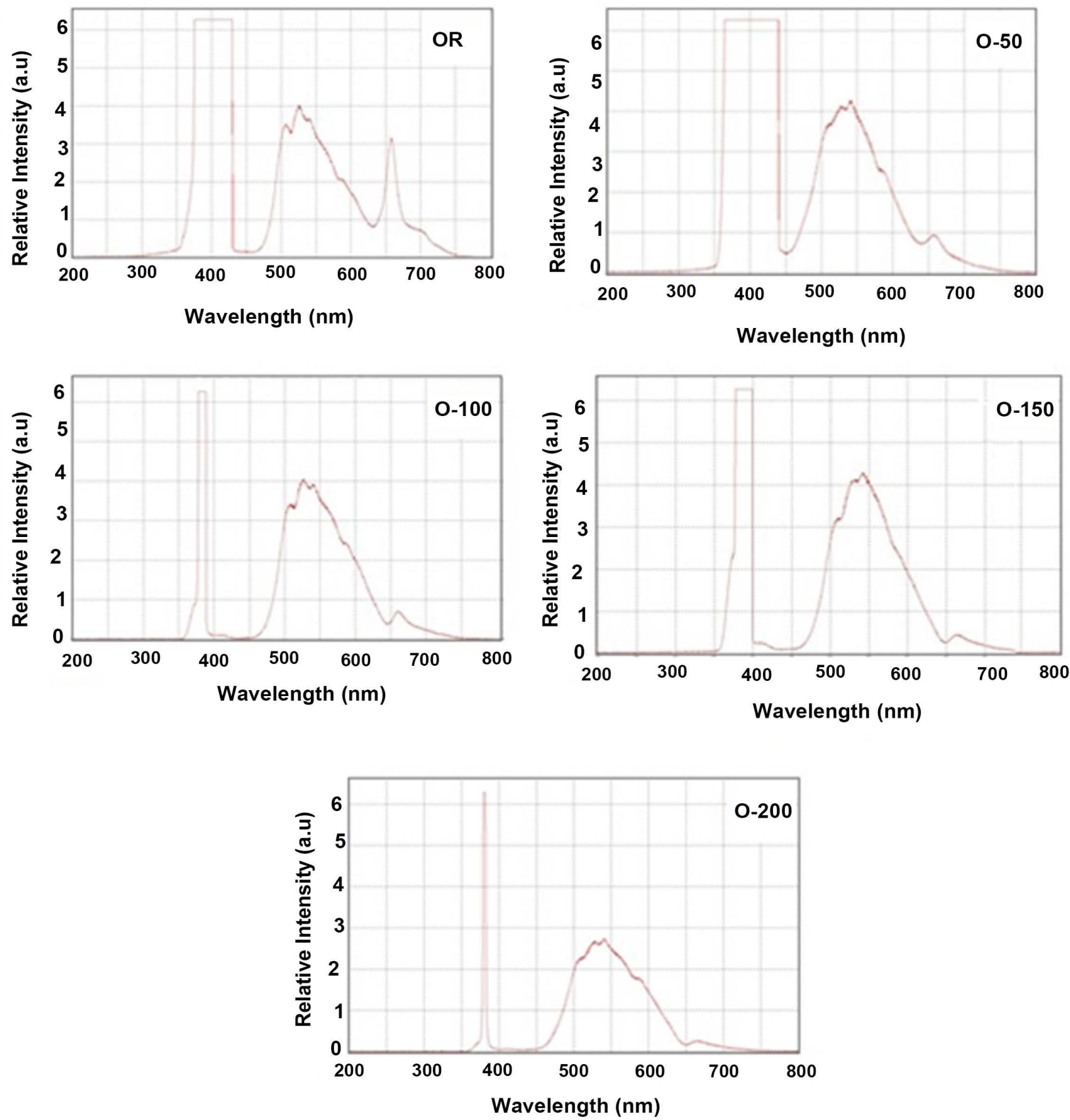

Figure 9. Laser-induced fluorescence spectra for EVOO at different temperatures.

two different brands of olive oils to study the heating effect and reported that tocopherols and polyphenols were the most affected by the thermal treatment with highest degradation rate. The degradation of vegetable oil quality is mainly caused by the oxidation of fatty acid, which produces hydroperoxide and some volatile compounds through initiation, propagation and termination [23]. The initiation phase starts with breakdown of hydrogen bond by the reaction

$$
R_{1}-\mathrm{CH}=\mathrm{CH}-\mathrm{R}_{2} \rightarrow \mathrm{R}_{1}-\mathrm{CH}=\dot{\mathrm{C}}-\mathrm{R}_{2}+\dot{H}
$$

Fatty acid Free radical 

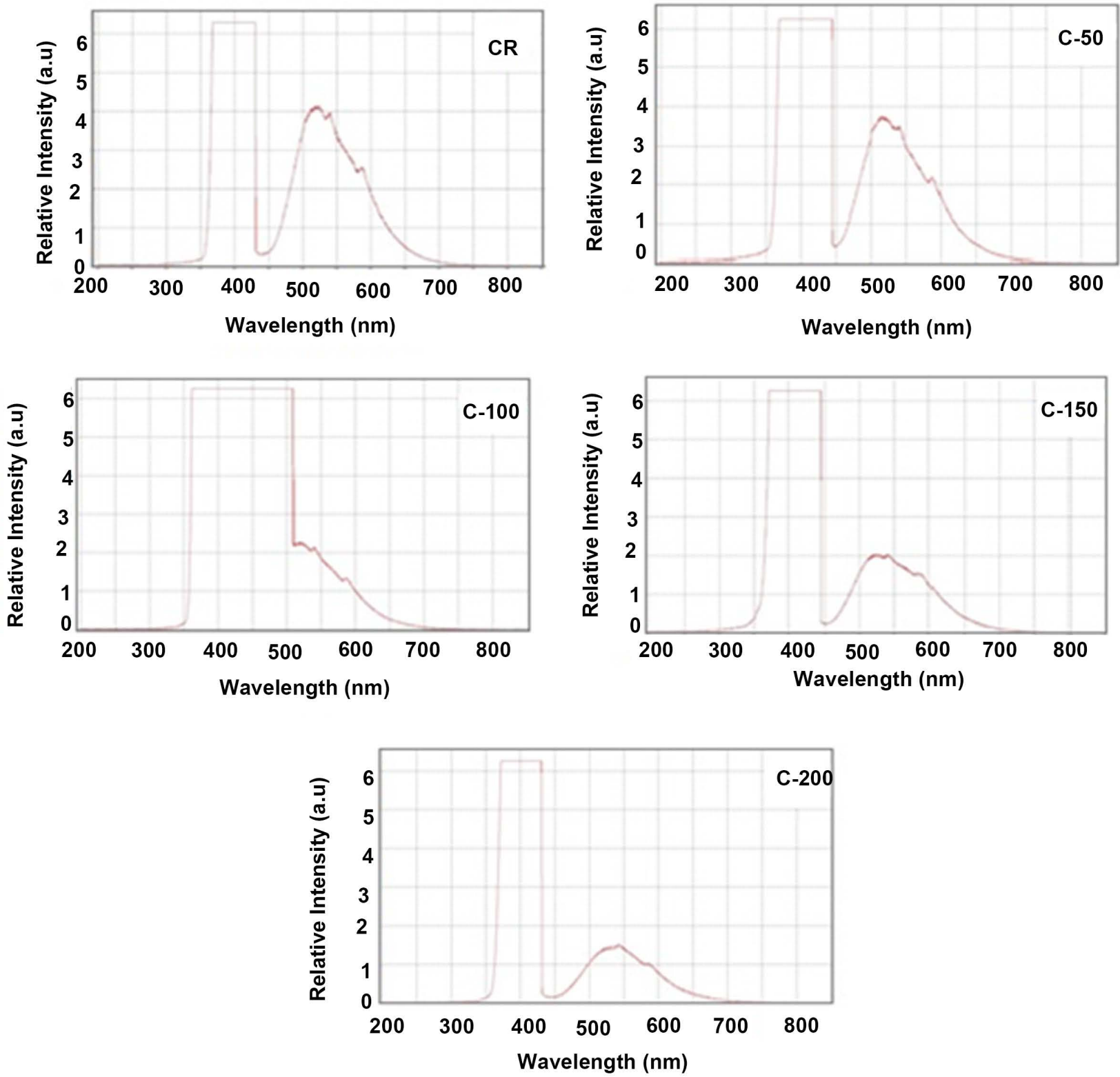

Figure 10. Laser-induced fluorescence spectra for $\mathrm{CO}$ at different temperatures.

where the initiator can be in the form of heat, light, free radicals, photosensitizing pigments and metal ions [23]. This reaction produces two free radicals that are responsible for producing peroxyradicals, which will grow rapidly in oxygen-rich sites. Consequently, hydrogen is taken out from an unsaturated fatty acid to form hydroperoxide and other free radical. The concentration of produced hydroperoxide increases during the propagation stage and at the termination phase, the oxidation process continuous the transformation of hydroperoxides into secondary nonradical oxidation compounds such as aldehydes, alcohols, esters, short-chain hydrocarbons and volatile ketones. Therefore, the presence of antioxidants including vitamin E, phenols and carotenoids in oils are essential to counteract the oxidation process. 
The results of UV-Vis absorption spectroscopy of both oils showed a systematic decrease of all dominant absorbance peaks with increase of temperature. This can be explained by Maxwellian distribution where by increasing the temperature, the thermal velocity distribution curve of molecules broadens and thus, the peak of curve is red-shifted. This red-shift in turn implies that the molecules no longer observe the optimized or maximum absorbing photons hence causing a change in peak. Therefore, the molecules population ends up absorbing a broader range of wavelengths than it did at a lower temperature. Indeed, the absorption process is very specific and results in an attenuation of the radiation and an increase of the electrons of the atom or molecules. Thus, such a shift will result in lowering the amount of energy absorption by specific target molecules. The root mean square velocity of molecules is

$$
\sqrt{\left\langle c^{2}\right\rangle}=\sqrt{\frac{3 R T}{M}}
$$

where $M$ is the molecular mass, $R=8.31 \mathrm{~J} \cdot \mathrm{mol}^{-1} \cdot \mathrm{K}^{-1} \cdot \mathrm{mol}^{-1}$ universal gas constant and $T$ is the temperature. For polyatomic system the average kinetic energy is

$$
\langle K \cdot E\rangle=3 / 2 K T
$$

which causes the energetic displacement of molecules with respect to their equilibrium position consequently results in more deviation of molecules from the beam path. Thus, effectively, we have

$$
\frac{\Delta \lambda}{\lambda_{0}}=\beta \frac{T}{M}
$$

where $\Delta \lambda$ is the change in incoming laser wavelength and $\lambda_{0}$ is the initial laser wavelength and $\beta$ is the proportionality constant. Such temperature-induced molecular displacement can also create more inter-molecular space within the medium through which the beam can propagate and be transmitted more then when it was at lower temperature, Figure 8. From Beer's Law

$$
A=-\log _{10} T=\varepsilon c l
$$

where $A$ is the absorbance of medium, $\varepsilon$ is the molar absorptivity $\left(\mathrm{M}^{-1} \cdot \mathrm{cm}^{-1}\right)$, c is the concentration and $l$ is the optical path length. Thus, by increasing the temperature absorbance decreases and consequently the transmittance increases which confirms the results in Figure 8. The deviation of the beam i.e., nonlinearity can be explained because of the refractive index of medium, which changes by temperature and the deviation of the beam occurs. The heating can produce thermal gradient within the oil due to absorption of light energy and redistribute the concentration of molecules. These factors can change the refractive index of medium. Therefore, thermal and concentration diffusion of molecules occur due to local heating by the laser beam inside the oil. It is noteworthy that $\Delta \mathrm{n}$ can be caused by both thermal and nonthermal effects where in the first case, the change of refractive index caused by thermal heating and concentration redistribution is given by 


$$
\frac{\mathrm{d} n(r, z)}{\mathrm{d} T}=\left(\frac{\partial n}{\partial T}\right)_{c}+\frac{\partial n}{\partial c} \frac{\partial c}{\partial T}
$$

In the case of EVOO included 425, 450 and $476 \mathrm{~nm}$ corresponding to presence of carotenoids with possible overlapping between $407 \mathrm{~nm}$ peak of pheophytin-a and $424 \mathrm{~nm}$ peak of carotenoid, vitamin E with a characteristic peakat $525 \mathrm{~nm}$ and the chlorophyll band with two maxima at 430 and $670 \mathrm{~nm}$. In the case of $\mathrm{CO}$, the peaks at 280, 360 and $420 \mathrm{~nm}$ mainly corresponding to linoleic and oleic acids exhibited a similar behaviour. During the heating in both cases, significant changes such as decrease of oxidative stability were observed above $100^{\circ} \mathrm{C}$, which can suggest the depletion of antioxidants. Oxidative stability is resistance of oils against oxidation during processing and storage [24] and is a key indicator to determine the quality of oil shell-life [25] because low-molecular weight off-flavour compounds are created during oxidation process. Therefore, essential fatty acids are destroyed, and some toxic compounds and oxidizing polymers are produced [26]. Deodorization, is a term used in industry to represent the processing stage where isomeration occurs from cis (i.e., the ends of carbon chain of fatty acid is bent towards each other) to trans (i.e., opposite direction). From a nutritional point of view the cis is more desirable as fatty acids with trans tends to modify the texture and melting properties of fat or oil. During refine processing of CO, formation of trans isomers of linolenic and linoleic acids are observed and oleic acid is less prone to isomeration [27]. It was found out that after two hours at 260 , about $22 \%$ of the linoleic acid was transformed into trans isomers. As it is seen from Figure 3 there is a significant change between the fresh and the aged EVOO. This can be explained by the fact that during storage, oil is subject to hydrolysis, oxidation and polymerization all of which result in deuteration of oil quality both in sensory and nutritive values. Hydrolysis can further undergo oxidation reactions, which was explained above [28]. All the possible changes occurring in an oil for different reasons exhibit the chemical alterations in different ways as observed in LIFS results where the amplitudes of fluorescence intensity progressively decreased in both EVOO and CO with temperature. For example, by increasing the temperature the peak corresponding to chlorophyll at $660 \mathrm{~nm}$ is strongly diminished and in fact vanishes at $\approx 200^{\circ} \mathrm{C}$ (Figure 9). Also, adulteration indicated a noticeable change in absorption spectrum and it can be utilized to test the originality, however, to differentiate between different types of oils LIFS can be employed simultaneously for the purpose of completeness.

\section{Conclusion}

UV-Vis absorption and fluorescence spectroscopy were used to test the quality and changes in the chemical structure of two types of EVOO and CO. The results confirmed that the composition and possible changes can be detected by both systems. The increase of temperature caused a change in the molecular structures of both types of oils seen as a gradual decrease of intensity amplitudes 
of absorption and fluorescence signals. In our case, a dramatic alteration occurred at $\approx 200^{\circ} \mathrm{C}$ where almost the main spectra of pheophytin-a, b, carotenoids, lutein and vitamin $\mathrm{E}$ in EVOO and linoleic acid and oleic acid in CO are disappeared. Aging and adulteration of an oil can also be tested by these systems and it is suggested best tested by using a combined system.

\section{Acknowledgements}

MEK is thankful to Mr Saeid Mohmedi the president of MIS Electronics Inc. for his support and funding the project and also Dr K. Deshmukh for her assistance and discussion.

\section{References}

[1] Garcia, A., Ruiz-Mendez, M. and Romero C. (2006) Effect of Refine on the Phenolic Composition of Crud Olive Oils. Journal of the American Oil Chemists' Society, 83, 159-164. https://doi.org/10.1007/s11746-006-1189-8

[2] Dabbou, S., Nakbi, A. and Noureddine, G. (2010) Compositional Quality of Virgin Oils from Cultivars Introduced in Tunisian Arid Zones in Comparison to Chemlali Cultivars. Scientia Horticulturae, 124, 122-127. https://doi.org/10.1016/j.scienta.2009.12.017

[3] Escrich, E., Ramírez-Tortosa, M., Sánchez-Rovira, P., et al. (2008) Olive Oil in Cancer Prevention and Progression. Nutrition Reviews, 64, S40-S52. https://doi.org/10.1111/j.1753-4887.2006.tb00262.x

[4] Bartoli, R. Fernandez-Banares, F., Navarro, E., et al. (2000) Effect of Olive Oil on Early and Late Events of Colon Carcinogenesis in Rats: Modulation of Arachidonic Acid Metabolism and Local Prostaglandin E2 Synthesis. Gut, 46,191-199. https://doi.org/10.1136/gut.46.2.191

[5] Gutiérrez, F., Arnaud T. and Garrido, A. (2001) Contribution of Polyphenols to the Oxidative Stability of Virgin Olive Oil. Journal of the Science of Food and Agriculture, 81, 1463-1470. https://doi.org/10.1002/jsfa.958

[6] Schneider, C. (2005) Chemistry and Biology of Vitamin E. Molecular Nutrition \& Food Research, 49, 7-30. https://doi.org/10.1002/mnfr.200400049

[7] El-Hamady, A. and El-Fizga, N. (1995) Detection of Olive Oil Adulteration by Measuring Its Authenticity Factor Using Reversed-Phase High Performance Liquid Chromatography. Journal of Chromatography A, 708, 351-355. https://doi.org/10.1016/0021-9673(95)00415-J

[8] Lopez-Diez, E., Bianchi, G., Goodacre, R., et al. (2003) Rapid Quantitative Assessment of the Adulteration of Virgion Olive Oils with Hazelnut Oils Using Raman Spectroscopy and Chemometrics. Journal of Agricultural and Food Chemistry, 51, 6145-6150. https://doi.org/10.1021/jf034493d

[9] Mata-Espinosa, P., Bosque-Sendra J, et al. (2008) Discriminating Olive and Non-Olive Oils Using HPLC-CAD and Chemometrics. Analytical and Bioanalytical Chemistry, 399, 2083-2092. https://doi.org/10.1007/s00216-010-4366-4

[10] Rohman, A. and Man, Y. (2010) Fourier Transform Infrared (FTIR) Spectroscopy for Analysis of Extra Virgin Oil Adulterated with Palm Oil. Food Research International, 43, 886-892. https://doi.org/10.1016/j.foodres.2009.12.006

[11] Girolamo, F., Masotti, SA., Lante, I, et al. (2015) A Simple and Effective Mass Spectrometric Approach to Identify the Adulteration of the Mediterranean Diet Com- 
ponent Extra Virgin Olive Oil with Corn Oil. International Journal of Molecular Sciences, 16, 20896-20912. https://doi.org/10.3390/ijms160920896

[12] Smejalova, D. and Piccolo, A. (2010) High-Power Gradient Diffusion NMR Spectroscopy for the Rapid Assessment of Extra-Virgin Olive Oil Adulteration. Food Chemistry, 118, 153-158. https://doi.org/10.1016/j.foodchem.2009.04.088

[13] Souza, R., Mathias, B., Silveira, C., et al. (2005) Inductively Coupled Plasma Optical Emission Spectrometry for Trace Multi-Element Determination in Vegetable Oils, Margarine and Butter after Stabilization with Propan-1-ol And Water. Spectrochimica Acta Part B: Atomic Spectroscopy, 60, 711-715. https://doi.org/10.1016/j.sab.2005.02.025

[14] Baeten, V., Meurens, M., Morales, M., et al. (1996) Detection of Virgin Olive Oil Adulteration by Fourier Transform Raman Spectroscopy. Journal of Agricultural and Food Chemistry, 44, 2225-2230. https://doi.org/10.1021/jf9600115

[15] Dankowska, A. and Malecka, M. (2009) Application of Synchronous Fluorescence Spectroscopy for Determination of Extra Virgin Olive Oil Adulteration. European Journal of Lipid Science and Technology, 223, 655-661. https://doi.org/10.1002/ejlt.200800295

[16] Zandomeneghi, M., Carbonaro, L. and Caffarata, Ch. (2005) Fluorescence of Vegetable Oils: Olive Oils. Journal of Agricultural and Food Chemistry, 53, 759-766. https://doi.org/10.1021/jf048742p

[17] Sikorska, E., Khmelinskii, I., Herance, I., et al. (2004) Characterization of Edible Oils Using Luminescence Spectroscopy. Journal of Fluorescence, 14, 25-35. https://doi.org/10.1023/B:JOFL.0000014656.75245.62

[18] Alfano, R., Tang, G., Pradhan, A., et al. (1987) Fluorescence Spectra from Cancerous and Normal Human Breast and Lung Tissues. IEEE Journal of Quantum Electronics, 23, 1806-1811. https://doi.org/10.1109/JQE.1987.1073234

[19] Khosroshahi, M.E. and Rahmani, M. (2011) Detection and Evaluation of Normal and Malignant Cells Using Laser-Induced Fluorescence Spectroscopy. Journal of Fluorescence, 22, 281-288. https://doi.org/10.1007/s10895-011-0958-4

[20] Domenici, V., Ancra, D., Cifelli, M., et al. (2014) Extraction of Pigment Information from Near UV-Vis Absorption Spectra of Extra Virgin Olive Oil. Journal of Agricultural and Food Chemistry, 62, 9317-9325. https://doi.org/10.1021/jf503818k

[21] Moneim Mahmoud, E., Dostalova, J., Pokorny, J., et al. (2009) Oxidation of Olive during Microwave and Conventional Heating for Fast Food Preparation. Czech Journal of Food Sciences, 27, S173-S177. https://doi.org/10.17221/963-CJFS

[22] Allouche, Y., jimenez, A., Gaforio, J., et al. (2007) How Heating Affects Extra Virgin Olive Oil Quality Indices and Chemical Composition. Journal of Agricultural and Food Chemistry, 55, 9646-9654. https://doi.org/10.1021/jf070628u

[23] Laguurre, J., Lecomte, J., Villenuve, P., et al. (2007) Evaluation of the Ability of Antioxidants to Counteract Lipid Oxidation: Existing New Methods. Progress in Lipid Research, 46, 244-282. https://doi.org/10.1016/j.plipres.2007.05.002

[24] Guillen, M.D. and Cabo, N. (2002) Fourier Transform Infrared Spectra Data versus Peroxide and Anisidine Values to Determine Oxidative Stability of Edible Oils. Food Chemistry, 77, 503-510. https://doi.org/10.1016/S0308-8146(01)00371-5

[25] Hamilton, R.J. (1994) The Chemistry of Rancidity in Foods. In: Allen, J.C. and Hamilton, R.J., Eds., Rancidity in Foods, 3rd Edition, Blackie Academic \& Professional, London, 1-21.

[26] Eunok, C. and Min, D. (2006) Mechanisms and Factors for Edible Oil Oxidation. 
Comprehensive Reviews in Food Science and Food Safety, 5, 169-171. https://doi.org/10.1111/j.1541-4337.2006.00009.x

[27] Mag, T.K. (1983) Canola Oil Processing in Canada. Journal of the American Oil Chemists' Society, 60, 380-384. https://doi.org/10.1007/BF02543522

[28] Gharby, S., Harhar, H., Mattuaus, B., et al. (2016) The Chemical Parameters and Oxidative Resistance to Heat Treatment of Refined and Extra Virgin Morocan Picholine Olive Oil. Journal of Taibah University for Science, 10, 100-106.

https://doi.org/10.1016/j.jtusci.2015.05.004 